Review Article

\title{
Potential Effects of Adjuvant Treatments on Coronavirus Disease 2019 (COVID- I9)
}

Tugolbai Tagaev', Melis Sholpanbai Uulu², Aizhamal Esengeldi Kyzy³, Vityala Yethindra", Elmira Mainazarova ${ }^{5}$, Sagynali Mamatov ${ }^{6}$, Altynai Zhumabekova ${ }^{7}$, Ashraf Syed Arslan ${ }^{8}$, Gaurav Singh Rathore ${ }^{9}$, Murtaza Afzal Lone ${ }^{10}$

${ }^{1}$ Department of Pathology, International Higher School of Medicine, International University of Kyrgyzstan, 1F, Intergelpo Street, Bishkek, 720054, Kyrgyzstan.

${ }^{2}$ Department of Infectious Diseases, I.K. Akhunbaev Kyrgyz State Medical Academy, 92, Akhunbaev Street, Bishkek, 720020, Kyrgyzstan.

${ }^{3}$ Department of Clinical Disciplines No. 1, Osh State University, 331, Lenin Street, Osh, 723500, Kyrgyzstan.

${ }^{4}$ International Higher School of Medicine, International University of Kyrgyzstan, 1F, Intergelpo Street, Bishkek, 720054, Kyrgyzstan. ${ }^{5}$ Department of Experimental Pathophysiology, Kyrgyz-Russian Slavic University, 44, Kiev Street, Bishkek, 720000, Kyrgyzstan. ${ }^{6}$ Department of Hospital Internal Medicine, Occupational Pathology with a Course of Hematology, I.K. Akhunbaev Kyrgyz State Medical Academy, 92, Akhunbaev street, Bishkek, 720020, Kyrgyzstan.

${ }^{7}$ Clinical Maternity Hospital No. 2, 225 Moskva Street, Bishkek, Kyrgyzstan, 720010.

${ }^{8,9,10}$ International Higher School of Medicine, International University of Kyrgyzstan, 1F, Intergelpo Street, Bishkek, 720054, Kyrgyzstan.

DOI: https://doi.org/10.24321/0019.5138.202022

I $\quad \mathbf{N} \quad \mathbf{F} \quad \mathbf{O}$

\section{Corresponding Author:}

Tugolbai Tagaev, Department of Pathology, International Higher School of Medicine, International University of Kyrgyzstan, 1F, Intergelpo Street, Bishkek, 720054, Kyrgyzstan. E-mail Id:

ttagaev22.kg@gmail.com

Orcid Id:

https://orcid.org/0000-0002-3102-6524

How to cite this article:

Tagaev T, Uulu MS, Kyzy AE, Yethindra V, Mainazarova E, Mamatov S et al. Potential Effects of Adjuvant Treatments on Coronavirus Disease 2019 (COVID-19). J Commun Dis 2020; 52(2): 46-49.

Date of Submission: 2020-05-27

Date of Acceptance: 2020-06-25

\section{$\begin{array}{llllllllllllll}\mathbf{A} & \mathbf{B} & \mathbf{S} & \mathbf{T} & \mathbf{R} & \mathbf{A} & \mathbf{C} & \mathbf{T}\end{array}$}

At present, there are no effective treatments for coronavirus disease 2019 (COVID-19) and the therapeutic interventions are limited to supportive care, varying from symptomatic management to critical care support. Corticoids are administered to reduce host inflammatory responses in the lungs. The potential harm and the lack of proven benefit of corticosteroids cautions against their routine administration in COVID-19 patients outside the Randomized Clinical Trials (RCTs), with an exception of a concomitant indication, such as a refractory shock. The amplified immune response and Cytokine Release Syndrome (CRS) are known to cause significant organ damage (lungs), providing a rationale for the use of monoclonal Antibody (mAb). Hyperimmune Immunoglobulins (Igs) from the recovered patients may also assist with improving the immune response and virus clearance. Human Normal Immunoglobulins (HNIGs) can be administered in the early seven to ten days of infection, during peak viremia or in the absence of a primary immune response. Ultimately, a vaccine could provide a potential permanent strategy, creating protective immunity for people and preventing future outbreaks.

Keywords: Coronavirus Disease 2019, Adjuvant Treatment, Corticosteroids, Monoclonal Antibody, Human Normal Immunoglobulins 


\section{Introduction}

Coronavirus disease 2019 (COVID-19) continues to affect more nations, leading to a possible economic and social collapse. Remdesivir, an antiviral drug originally developed to treat Ebola, showed inhibitory activity against Severe Acute Respiratory Syndrome Coronavirus (SARS-CoV) and Middle-East Respiratory Syndrome Coronavirus (MERSCoV) infections which affect the epithelial cells of the human respiratory-tract, by inhibiting viral RNA synthesis and therefore hindering the early stages of replication. ${ }^{1}$

At present, there are no effective antiviral drugs which would effectively target the viral attachment, its entry into the host cells, or genomic replication. It is clear that usage of potent therapeutics is the only option to decrease the COVID-19 morbidity and mortality before the vaccine becomes available; however, recently, patient therapy is mostly limited to supportive care, varying from a symptomatic management to critical care support. This article reviews three adjuvant treatments for COVID-19 that may have a promising therapeutic potential: corticoids, monoclonal Antibodies (mAb), and human normal immunoglobulin (HNIG).

\section{Corticoids}

Corticoids (glucocorticoids) are a class of steroid hormones commonly used to suppress inflammation. Glucocorticoids reversibly inhibit phospholipase $A 2$ and reduce the synthesis of cyclooxygenase and lipoxygenase enzymes, resulting in decreased production of pro-inflammatory cytokines, such as prostaglandins and leukotrienes. In COVID-19 patients, corticoids are administered to reduce host inflammatory responses in the lungs to avert the progress of Acute Respiratory Distress Syndrome (ARDS); however, these patients may develop side effects, such as decreased viral clearance and increased susceptibility to secondary infections. Studies on patients with SARS and MERS reported no associations between corticoids and improved survival. Furthermore, there was a decreased viral clearance in the lower respiratory tract and blood of these patients, as well as complications, such as hyperglycemia and avascular necrosis. ${ }^{2,3}$

Moreover, 10 studies involving 6,548 influenza pneumonia patients demonstrated that corticoids were associated with an elevated risk of death and a higher risk of secondary infections. ${ }^{4}$ Patients with bacterial, rather than viral, infections are benefitted from corticoid use. ${ }^{5}$

In a separate study in China, 201 COVID-19 patients, who developed ARDS, treated with methylprednisolone showed less fatality rate ( $46 \%$ with steroid treatment vs. $62 \%$ without steroids). ${ }^{6}$ Therefore, due to the potential harm and the lack of proven benefit of corticoids cautions against their routine administration in COVID-19 patients outside the Randomized Clinical Trials (RCTs), with an exception of a concomitant indication, such as a refractory shock.

\section{Monoclonal Antibodies}

Monoclonal antibodies (mAbs) targeting key inflammatory cytokines are another potential adjuvant treatment for COVID-19. Furthermore, an in vitro study using a neutralizing human mAb against SARS-CoV-2 showed promising results by blocking infection. ${ }^{7}$ It has been consistently documented that some patients with COVID-19 experience an amplified immune response and Cytokine Release Syndrome (CRS) in response to the virus, ${ }^{8}$ resulting in significant organ damage. Interleukin-6 (IL-6) has been shown to play a main role in this dysregulated inflammation. ${ }^{9}$ Therefore, using mAbs to target the inflammatory cytokines, such as IL-6, may decrease this severe reaction and improve clinical outcomes. For example, tocilizumab, a mAb against the IL-6 receptor, used for treating Rheumatoid Arthritis (RA) and CRS.

As a proof of this concept, the efficacy of tocilizumab was assessed in a less amount of severe COVID-19 cases and it showed promising outcomes..$^{10}$ In a study treatment with $400 \mathrm{mg}$ tocilizumab in 21 COVID-19 patients showed symptom amelioration in $91 \%$ of the patients, who are receiving a single dose. ${ }^{9}$ Sarilumab, the human $\mathrm{mAb}$ against the IL-6 receptor, is presently being analysed for hospitalized severe COVID-19 patients. ${ }^{11}$

The other mAbs being tested in RCTs are bevacizumab (antivascular endothelial growth factor medication), fingolimod (immunomodulator approved for multiple sclerosis), and eculizumab (an antibody inhibiting terminal complement). ${ }^{12}$

\section{Human Normal Immunoglobulin (HNIG) or Immunoglobulin (Ig) Therapy}

Hyperimmune Igs from the recovered patients are helpful in the immune response and viral clearance. In SARS and MERS outbreaks, Hyperimmune Igs was prescribed as a rescue therapy. ${ }^{13,14}$ In a 2009 prospective observational study involving critically ill H1N1 influenza A patients, 20 of the 93 patients received convalescent plasma and the treatment resulted in reduced mortality. ${ }^{15}$

In analysis of eight studies of 714 patients infected with SARS and treated with hyperimmune Ig reported reduced patient mortality and a less side effects, although the authors did report a moderate to high risk of bias. ${ }^{16}$ Administration of HNIGs appears to be the most advantageous in the early seven to ten days of infection, especially during peak viremia or absence of primary immune response. Furthermore, in China, the case studies of five and three critically ill COVID-19 patients administered with intravenous Ig for five days with a dose of 0.3 to $0.5 \mathrm{~g} / \mathrm{kg} /$ day showed good results. ${ }^{17,18}$ 
Although current commercial Ig preparations likely lack protective antibodies against SARS-CoV-2, this type of treatment warrants further safety and efficacy trials as the pool of patients infected by COVID-19 is increasing globally, a potential permanent strategy could be the advancement of a vaccine which provides immunity to people, thus preventing future outbreaks.

\section{Conclusion}

The accurate diagnosis and treatment of COVID-219 is of critical importance as it allows professionals to implement effective measures required to control and prevent its spread. This study reviews the potential of three therapeutic approaches for the treatment of COVID-19-corticoids, monoclonal antibodies, and human normal immunoglobulin. As per our review, both monoclonal antibodies and human normal immunoglobulin show promising potential as therapeutic strategies against COVID-19.

Although the advancement of a vaccine against COVID-19 is likely to take some time, careful measures are necessary, for now, to direct contact transmission of SARS-CoV-2. Laboratory screening and social isolation of potentially infected individuals before the clinical symptoms onset would diminish the disease spread. Individuals diagnosed with active infection accompanied by overt clinical manifestations must be isolated and treated in a hospital setting.

\section{Author Contributions}

Conception or design of the work: Tugolbai Tagaev.

Interpretation of data for the work: Elmira Mainazarova, Sagynali Mamatov, Gaurav Singh Rathore, Murtaza Lone.

Drafting the work: Sagynali Mamatov, Vityala Yethindra, Murtaza Lone.

Critical revised for important intellectual content: Tugolbai Tagaev, Melis Sholpanbai Uulu, Aizhamal Esengeldi Kyzy, Vityala Yethindra.

Final approval of the version to be published: Tugolbai Tagaev, Melis Sholpanbai Uulu, Aizhamal Esengeldi Kyzy, Vityala Yethindra, Elmira Mainazarova, Sagynali Mamatov, Gaurav Singh Rathore, Murtaza Lone.

\section{Sources of Funding: Not Applicable \\ Conflicts of Interest: None}

\section{References}

1. Yethindra V. Role of GS-5734 (Remdesivir) in inhibiting SARS-CoV and MERS-CoV: The expected role of GS5734 (Remdesivir) in COVID-19 (2019-nCoV) - VYTR hypothesis. Int J Res Pharm Sci 2020; 11: 1-6. DOI. org/10.26452/ijrps.v11iSPL1.1973.

2. Stockman LJ, Bellamy R, Garner P. SARS: systematic review of treatment effects. PLoS Med 2006; 3(9): e343. DOI: 10.1371/journal.pmed.0030343.

3. Arabi YM, Mandourah Y, Al-Hameed F, Sindi AA, Almekhlafi GA, Hussein MA et al . Saudi Critical Care Trial Group. Corticosteroid therapy for critically ill patients with Middle East respiratory syndrome. Am J Respir Crit Care Med 2018; 197(6): 757-767.

4. Ni YN, Chen G, Sun J, Liang BM, Liang ZA. The effect of corticosteroids on mortality of patients with influenza pneumonia: a systematic review and meta-analysis. Crit Care 2019; 23(1): 99. DOI: 10.1186/s13054-019-2395-8.

5. Russell CD, Millar JE, Baillie JK. Clinical evidence does not support corticosteroid treatment for 2019-nCoV lung injury. Lancet 2020; 395(10223): 473-475.

6. Wu C, Chen X, Cai Y et al. Risk Factors Associated With Acute Respiratory Distress Syndrome and Death in Patients With Coronavirus Disease 2019 Pneumonia in Wuhan, China. JAMA Intern Med 2020;e200994. doi:10.1001/jamainternmed.2020.0994.

7. Wang C, Li W, Drabek D, Okba NMA, Haperen RV, Osterhaus ADME et al. A human monoclonal antibody blocking SARS-CoV-2 infection. Nat Commun 2020; 11 : 2251. DOi: 10.1038/s41467-020-16256-y.

8. Mehta P, McAuley DF, Brown M, Sanchez E, Tattersall RS, Manson JJ. HLH Across Speciality Collaboration, UK. COVID-19: consider cytokine storm syndromes and immunosuppression. Lancet 2020; 395(10229): 1033-1034.

9. Zhou F, Yu T, Du R, Fan G, Liu Y, Liu Z et al. Clinical course and risk factors for mortality of adult in patients with COVID-19 in Wuhan, China: a retrospective cohort study. Lancet 2020; 395(10229): 1054-1062.

10. Xu X, Han M, Li T, Sun W, Wang D, Fuet B et al. Effective treatment of severe COVID-19 patients with tocilizumab. Proc Natl Acad Sci U S A 2020; 117(20): 10970-10975.

11. Sanofi. Sanofi and Regeneron begin global Kevzara (sarilumab) clinical trial program in patients with severe COVID-19 [news release]. Published March 16, 2020. Accessed March 18, 2020. http://www.news.sanofi. us/2020-03-16-Sanofi-andRegeneron-begin-globalKevzara-R-sarilumabclinical-trial-program-in-patientswith-severeCOVID-19.

12. ClinicalTrials.gov. Accessed March 18, 2020. https:// clinicaltrials.gov/.

13. Soo YO, Cheng Y, Wong R, Hui DS, Lee CK, Tsang KKS, et al. Retrospective comparison of convalescent plasma with continuing high-dose methylprednisolone treatment in SARS patients. Clin Micro Biol Infect 2004; 10(7): 676-678.

14. Arabi $Y$, Balkhy $H$, Hajeer $A H$, Bouchama A, Hayden FG, Al-Omari A et al. Feasibility, safety, clinical, and laboratory effects of convalescent plasma therapy 
for patients with Middle East respiratory syndrome coronavirus infection: a study protocol. Springerplus 2015; 4: 709. DOI: 10.1186/s40064-015-1490-9.

15. Hung IF, To KK, Lee CK, Lee KL, Chan K, Yan WW et al. Convalescent plasma treatment reduced mortality in patients with severe pandemic influenza A (H1N1) 2009 virus infection. Clin Infect Dis 2011; 52(4): 447-456.

16. Mair-Jenkins J, Saavedra-Campos M, Baillie JK, Cleary P, Khaw FM, Lim WS et al. Convalescent Plasma Study Group. The effectiveness of convalescent plasma and hyperimmune immunoglobulin for the treatment of severe acute respiratory infections of viral etiology: a systematic review and exploratory meta-analysis. J Infect Dis 2015; 211(1): 80-90.

17. Shen C, Wang Z, Zhao F, Yang Y, Li J, Yuan J et al. Treatment of 5 critically ill patients with COVID-19 with convalescent plasma. JAMA 2020; 323(16): 1582-1589.

18. Cao W, Liu X, Bai T, Fan H, Hong K, Song $\mathrm{H}$ et al. Highdose intravenous immunoglobulin as a therapeutic option for deteriorating patients with coronavirus disease 2019. Open Forum Infect Dis 2020; 7(3): ofaa102. DOI: 10.1093/ofid/ofaa102. 